\title{
Regulation and characterization of rot transcription in Staphylococcus aureus
}

Correspondence
Adhar C. Manna
amanna@usd.edu

Received 13 November 2006

Revised 15 January 2007

Accepted 21 January 2007

\author{
Adhar C. Manna and Binata Ray \\ Division of Basic Biomedical Sciences, University of South Dakota, Vermillion, SD 57069, USA, \\ and Center for Infectious Disease Research and Vaccinology, South Dakota State University, \\ Brookings, SD 57007, USA
}

\begin{abstract}
The pathogenesis of Staphylococcus aureus infections is dependent upon expression of various virulence factors, which are under the control of multiple regulatory systems, including two-component regulatory systems and transcriptional regulators such as the SarA family of proteins. As a part of a continuing effort to understand the regulatory mechanisms that involve the SarA protein family, the regulation and physical characterization of rot transcription is described here. The rot gene, a member of the sarA family of genes, was previously characterized and has been shown to regulate a large number of genes. The rot locus is composed of multiple overlapping transcripts as determined by primer extension and was proposed to encode an open reading frame of 133 residues. Transcription of rot was significantly increased in the sarA mutant. Gel shift and transcriptional studies revealed that SarA could bind to the rot promoter region, probably acting as a repressor for rot transcription. The data indicate that the expression of rot transcription is significantly repressed only by SarA among the sarA family of mutants tested at the post-exponential phase of growth.
\end{abstract}

\section{INTRODUCTION}

Staphylococcus aureus is a major pathogen in both community and hospital settings, and continues to be a serious public concern due to the lack of an effective vaccine and the emergence of multiple antibiotic-resistant strains (Arvidson \& Tegmark, 2001; Boyce, 1997; Lowy, 1998). The spectrum of diseases caused by this organism is extremely broad, ranging from localized skin suppuration to life-threatening septicaemia. Despite antimicrobial therapy, morbidity and mortality associated with $S$. aureus infections remains high, due, in part, to the organism's ability to develop resistance to virtually all antibiotics, including vancomycin (Arvidson \& Tegmark, 2001; Lowy, 1998). Within infection sites, S. aureus produces several virulence factors that include surfaceassociated proteins, enzymes, exotoxins, capsular polysaccharides and gene products that facilitate tissue colonization, tissue destruction and immune evasion. The expression of many of these virulence genes is coordinately controlled by regulatory loci, such as two-component regulatory systems (agr, saeRS, srrAB and arlSR) and global transcriptional regulators (sarA, sigB, sarA paralogues, tcaRA, etc.) (Arvidson \& Tegmark, 2001; Cheung et al., 1992; Cheung \& Zhang, 2002; Luong et al., 2003; Manna \& Cheung, 2001, 2003, 2006a, b; Manna et al., 2004; McNamara et al., 2000; McNamara \& Bayer, 2005; Novick, 2003; Projan \& Novick, 1997; Truong-Bolduc et al., 2003).

The sarA locus, a well-studied transcriptional regulator in $S$. aureus, is important for the regulation of various genes involved in virulence, regulatory and metabolic processes (Bayer et al., 1996; Cheung et al., 1992; Chien \& Cheung, 1998; Chien et al., 1999; Dunman et al., 2001). The sarA locus consists of a major open reading frame, SarA (124 residues), driven by three distinct promoters, resulting in three overlapping transcripts with a common terminating end (Bayer et al., 1996). The sarA locus regulates the expression of selected cell-wall-associated proteins and exoproteins (e.g. $\alpha$ - and $\beta$-haemolysins). SarA binds to several target gene promoter regions including regulatory systems such as agr, sarS and sarV (Cheung et al., 2001; Chien \& Cheung, 1998; Manna et al., 2004; Rechtin et al., 1999) and virulence genes such as hla, spa, cna, bap, ica and fnbA to modulate gene transcription (Blevins et al., 1999; Chien et al., 1999; Tormo et al., 2005) by both direct and indirect pathways. Microarray analyses demonstrated that a sarA mutation altered the expression of over 120 genes, with 76 upregulated and 44 downregulated (Dunman et al., 2001).

Analysis of published S. aureus genomes reveals that at least 10 major SarA paralogues are present (Kuroda et al., 2001). SarA is the first characterized member of the SarA family; others characterized are SarR (Manna \& Cheung, 2001), SarS (also called SarH1) (Cheung et al., 2001; Tegmark et al., 2000), SarT (Schmidt et al., 2001), Rot (McNamara et al., 2000), SarU (Manna \& Cheung, 2003), SarV (Manna et al., 2004), MgrA (Luong et al., 2003; Truong-Bolduc et al., 2003) and SarX (Manna \& Cheung, 2006b). The SarA family 
represents a group of DNA-binding proteins that share homology with each other (20-45\% identity and 45-65\% similarity to SarA protein) as well as with the MarR family of proteins involved in regulating multi-drug resistance in Gram-negative bacteria (Cheung \& Zhang, 2002). Although some members of the sarA family of genes are only partially characterized, the known attributes of nine sarA-like genes suggest that most SarA paralogues function as both repressors and activators. They regulate one another and other regulatory and target genes either by binding directly to promoter regions or by acting indirectly via other regulators. Structural analysis suggests that smaller SarA proteins exist as dimers (e.g. SarA, SarR, MgrA), while the larger proteins with two homologous domains are monomers (e.g. SarS) and are also homologous to the wingedhelix family of transcription factors with unique variations (Cheung \& Zhang, 2002; Liu et al., 2001, 2006; Manna \& Cheung, 2006a). While many of these genes either activate or repress the expression of virulence and regulatory genes, the exact mode of regulation is not well defined.

The rot gene, a member of the sarA family of genes, has been characterized and is suggested to be a global regulator in $S$. aureus. Rot, a 166-residue SarA paralogue (or 153-residue as predicted in published $S$. aureus genomes) represses haemolysins ( hla and hld), toxin and lipase (geh), and positively regulates cell-surface-associated protein synthesis [clumping factor, protein A, putative cell-surface adhesin $(s d r C)$ ] (McNamara et al., 2000; McNamara \& Bayer, 2005; Said-Salim et al., 2003). Transcriptional profiling suggests that inactivation of rot affects the transcription of 168 genes in $S$. aureus including sarS, another member of the sarA family of genes (Said-Salim et al., 2003). Although rot has been shown to be a global regulator for virulence gene expression, the regulation of rot transcription, particularly by other members of the sarA family of genes, is not known and physical mapping of rot transcript has not been performed. However, recently it has been shown that the translation of rot transcript is inhibited by the agr RNAIII molecule (Geisinger et al., 2006).

In our continuing effort to understand the underlying mechanism by which the SarA protein family functions at various levels, we report here the regulation of expression of rot transcription by other known sarA family mutants and the physical mapping of the rot transcripts.

\section{METHODS}

Bacterial strains and growth conditions. The bacterial strains and plasmids used in this study are described in the text. Routinely used standard Escherichia coli DH5 $\alpha$ and BL21(DE3) strains were employed for manipulation of DNA and overexpression of protein. Plasmid DNA manipulated in E. coli was transferred to the restriction-deficient S. aureus strain RN4220 by electroporation (Schenk \& Laddaga, 1992). S. aureus cells were grown at $37^{\circ} \mathrm{C}$ with constant aeration (220 r.p.m.) in tryptic soy broth (TSB) or on tryptic soy agar (TSA) supplemented with antibiotics when necessary. LuriaBertani (LB) medium was used for growing E. coli. When required, antibiotics were added to the culture media in the following concentrations: for S. aureus, $5 \mu \mathrm{g}$ erythromycin $\mathrm{ml}^{-1}, 3 \mu \mathrm{g}$ tetracycline $\mathrm{ml}^{-1}$, and $10 \mu \mathrm{g}$ chloramphenicol $\mathrm{ml}^{-1}$, or for E. coli, $50 \mu \mathrm{g}$ ampicillin $\mathrm{ml}^{-1}$ and $30 \mu \mathrm{g}$ chloramphenicol $\mathrm{ml}^{-1}$.

Genetic manipulation in E. coli and S. aureus. Construction and characterization of most of the mutants was published earlier (Manna \& Cheung, 2001, 2006b), except for the sarZ (SA2174 of $S$. aureus $\mathrm{N} 315$ ) mutant (unpublished).

Isolation of RNA and Northern blot hybridization. Total RNA isolation from different $S$. aureus strains and Northern blot hybridization were performed as previously described (Bayer et al., 1996; Manna \& Cheung, 2001).

Transcriptional fusion studies of rot promoter linked to the gfp $_{\text {uvr }}$ reporter gene. A $317 \mathrm{bp}$ rot promoter fragment (nt 204-520; GenBank accession no. AF189239 or positioned from +1 to $317 \mathrm{bp}$ of the rot promoter) (see Fig. 2) containing the upstream promoter region of the rot gene was amplified by PCR, using chromosomal DNA of $S$. aureus strain RN6390 and primers with flanking EcoRI or $X b a \mathrm{I}$ sites. An EcoRI and $\mathrm{XbaI}$ fragment containing the rot promoter region was cloned into shuttle plasmid pALC1484, generating a transcriptional fusion of the rot promoter to the $g f p_{u v r}$ reporter gene (Kahl et al., 2000). Restriction analysis and DNA sequencing confirmed the orientation and authenticity of the promoter reporter gene construct. The recombinant plasmid containing the rot promoter region and a promoterless plasmid as a control were first introduced into $S$. aureus strain RN4220 by electroporation. Plasmids purified from RN4220 transformants were then electroporated into RN6390, its various isogenic mutants, and single-copy complemented sarA (ALC812) strains as required.

After overnight incubation, $S$. aureus strains harbouring the recombinant plasmids were diluted $1: 100$ in TSB containing chloramphenicol and grown at $37^{\circ} \mathrm{C}$ with shaking at 220 r.p.m. Aliquots $(200 \mu \mathrm{l})$ were transferred in triplicate hourly for $10 \mathrm{~h}$ to microtitre plates to assay for $\mathrm{OD}_{650}$ and green fluorescent protein (GFP) fluorescence with an FL800TB1 fluorescence reader (BioTek Instruments). Promoter activity was plotted as mean fluorescence per $\mathrm{OD}_{650}$ over time, using the mean values from triplicate readings calculated by subtracting the values for promoterless control.

Purification of SarA protein and gel shift analysis. The cloning and purification of $\mathrm{His}_{6}$-SarA protein were described previously (Chien et al., 1999). The purity of His fusion SarA protein was checked in a $12 \%$ polyacrylamide-SDS gel and found to be more than $98 \%$ pure. The concentration of the purified protein was determined by using the Bio-Rad protein estimation kit and BSA as the standard.

In order to determine if the recombinant SarA protein binds to the rot promoter region, a $317 \mathrm{bp}$ fragment used for transcriptional fusion studies, representing the rot promoters region, was end-labelled with $\left[\gamma^{32} \mathrm{P}\right] \mathrm{ATP}$ by using T4 polynucleotide kinase. The labelled fragment $(0.1 \mathrm{ng})$ was incubated at room temperature for $20 \mathrm{~min}$ with various amounts of purified SarA protein in $25 \mu \mathrm{l}$ binding buffer $(25 \mathrm{mM}$ Tris/ $\mathrm{HCl}, \mathrm{pH} 7.5,0.1 \mathrm{mM}$ EDTA, $75 \mathrm{mM} \mathrm{NaCl}, 1 \mathrm{mM}$ dithiothreitol and $5 \%$ glycerol) containing $0.5 \mu \mathrm{g}$ calf thymus DNA (Invitrogen). The reaction mixtures were analysed in an $8.0 \%$ non-denaturing polyacrylamide gel. The band shifts were detected by exposing dried gels to X-ray films.

Primer extension analysis. Mapping of the $5^{\prime}$ end of the rot transcripts by primer extension was performed by using the primer $5^{\prime}-$ ATTCGCTTTCAATCTCGCTGAAAATT-3', complementary to the rot coding strand and located from nucleotide positions 72 to 97 downstream from the putative proposed start codon ATG (nt 


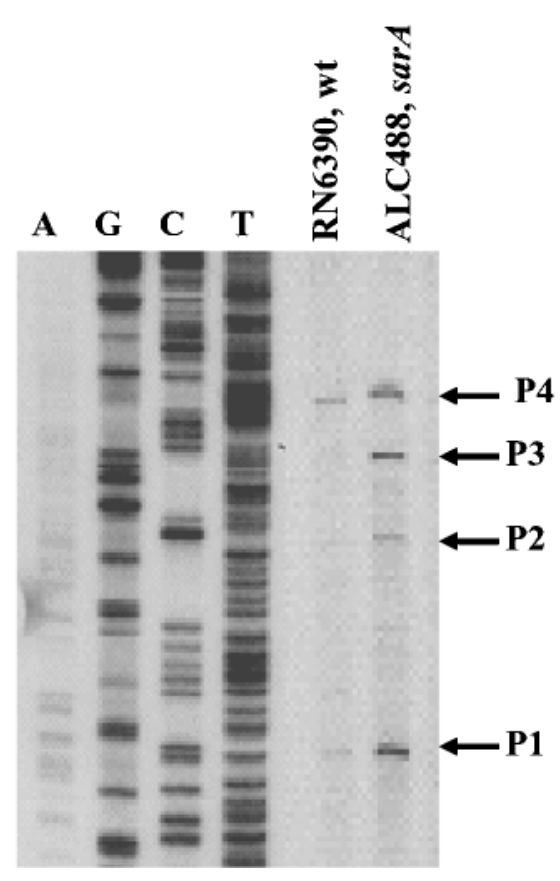

Fig. 1. Promoter analysis of the rot gene in S. aureus. Primer extension of the rot transcripts by using total RNA isolated from the wild-type RN6390 and the sarA mutant strains at the postexponential phase of growth. P1, P2, P3 and P4 are designated according to the smallest length to the largest length of rot transcripts.

626-651; GenBank accession no. AF189239). Primer extension was carried out by using $30 \mu \mathrm{g}$ total RNA isolated from wild-type RN6390 and sarA mutant strains in post-exponential-phase culture as described elsewhere (Ausubel et al., 2000; Bayer et al., 1996). The same radiolabelled primer was used for the DNA sequencing reactions.

\section{RESULTS}

\section{Characterization of the rot transcripts}

Although the phenotypic characterization of the rot gene has been performed thoroughly, the regulation and characterization of rot transcription are not well defined (McNamara et al., 2000; McNamara \& Bayer, 2005; Said-Salim et al., 2003; Tseng \& Stewart, 2005). Therefore, in order to determine the transcriptional start site and the promoter structure of the rot transcript and the probable length of the rot open reading frame, primer extension analysis was performed with total RNA isolated from the wild-type RN6390 and sarA mutant strains (Fig. 1). The primer extension results were interesting between the two isogenic strains analysed as two primer extension bands (designated $\mathrm{P} 1$ and $\mathrm{P} 4$ ) were detected in the case of the wild-type strain, and four bands (designated P1, P2, P3 and P4) were detected in the case of the sarA mutant. A significant elevation of P1 and $\mathrm{P} 4$ transcript signals was also observed in the case of the sarA mutant as compared to the wild-type strain, which indicates the probable role of sarA in regulating rot transcription. The transcriptional start sites of all four transcripts were mapped to 105, 172, 212 and $255 \mathrm{nt}$ downstream from the proposed Rot (133-residue) initiation codon ATG (Fig. 2), respectively. Based upon the transcriptional start sites, the predicted putative promoter regions $(-35$ and -10$)$ are shown in Fig. 2; they have close homology with the E. coli -35 and -10 consensus sequences (TTGACA- $\mathrm{N}_{14-21}$-TATAAT) of $\sigma^{\mathrm{A}}$-dependent promoters. The published translational start codon (ATG) for 166-residue Rot was located at the $+7 \mathrm{nt}$ position from the mapped transcriptional start site +1 of the smallest transcript $\mathrm{P} 1$ and the region lacked any defined ribosomebinding sequences (RBS). These results suggest that the rot open reading frame may encode less than a 166-residue polypeptide. Secondly, the computer-generated previously published translational start codon ATG for Rot was located
1

ATATGTAATA AAAATTCAAT GCGTATCTT T TTTGAAGAAA TATATGTAGA ATTGTTGCAA TATACATTAT TTTTAAGTTA CG CATAGAAA AAACTTCTTT ATATACATCT TAACAACGTT

$\begin{array}{llllllll}61 & -35 & B 1 & P 4 & -10 & B 2 & +1 & -35\end{array}$

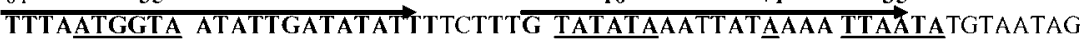
AAATTACCAT TATAACTATA TAAAAGAAAC ATATATTTAA TATTTT AATT ATACATTATC $\begin{array}{llllll}121 & \mathbf{P 3} & -10 & +1 & -35 & \mathbf{P} 2\end{array}$ B4

AGTGATTTGT TTTATGTACTA TTATCTTAT TTCTAAATATT AACTCTATT GATTATTGGT TCACTAAACAAAATACATGAT AATAGAATA AAGATTTATAATTGAGATAA CTAATAACCA

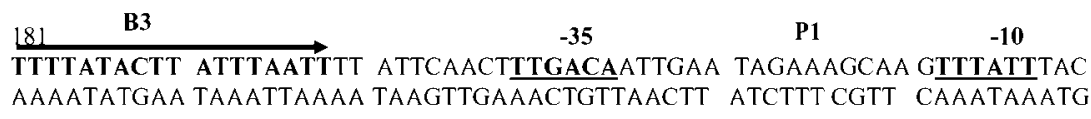
$241+1$ Rot 166 aa Rot 153 aa ACTTGT GGT TTATGCATAA GTTAGCACAT ACAAGTTTTGGGATTGTTGG GATGTTTGTT TGAACATCAA AATACGTATT CAATCGTGTA TGTTCAAAAC CCTAACAACC CTACAAACAA 301 RBS Rot 133 aa AATACTTGTA TGGTAGCTAA ATATGTGATT ATTAATTGGG AGATGTTTAG CATG ... TTA TGAACAT ACCATCGATT TATACACTAA TAATTAACCC TCTACAAATC GTAC
Fig. 2. Nucleotide sequence of the $354 \mathrm{bp}$ promoter region of the rot gene; putative promoter recognition sites (-35 and -10) are bold and underlined. The transcriptional start sites $(+1)$ were identified based on a primer extension and are in bold and underlined. The ribosome-binding sequences (RBS) and the putative predicted translational start codon (ATG) in various $S$. aureus genomes are marked in bold. The putative consensus SarA-binding regions (B1 to B4) on the promoter are marked by bold and arrows. 
at the $+46 \mathrm{nt}$ position from the mapped transcriptional start site of the P1 transcript and its adjacent region displays a relatively weak putative RBS (TTGGGATT). However, a strong RBS, GGGAGA, is located at the +92 nt position from the transcriptional start site of the P1 transcript. Based on the mapped transcriptional start sites and the presence of a strong putative RBS, we suggest that the ATG located at the +92 nt position (position 352; Fig. 2) is probably the Rot translational start codon and that Rot is a 133-residue polypeptide. Indeed, recent transcriptional fusion analysis of the upstream promoter region of the rot gene suggested that translation of rot initiated from the ATG codon located at the $+92 \mathrm{nt}$ position (Geisinger et al., 2006). Analysis of the downstream sequence of the rot gene disclosed the presence of a potential hairpin structure or rho-independent transcriptional terminator sequence from a position 13-48 bp immediately after the stop codon (TAA).

To further confirm, we affinity-purified approximately $16 \mathrm{kDa}$ Rot protein from a sarA mutant harbouring pSK236 plasmid carrying a $780 \mathrm{bp}$ DNA fragment, which contains a $350 \mathrm{bp}$ upstream sequence of the rot gene plus rot coding region with six histidine codons inserted before the stop codon. MALDI-TOF-MS and N-terminal amino acid sequencing verified the authentication of the purified Rot$\mathrm{His}_{6}$ protein band and the starting amino acid methionine $(\mathrm{M})$ at the 34-residue position (data not shown). This result was supported by the observation that the $15.6 \mathrm{kDa}$ Rot protein (corresponding to 133 residues) co-eluted with SarA, MgrA and SarR in an affinity pull-down assay from whole-cell lysates with a spa promoter fragment (Oscarsson et al., 2005). In addition, studies by Geisinger et al. (2006) with the C-terminal histidine fusion of the rot gene in a multicopy plasmid and immunoblot analysis with antihistidine antibody indicated the production of a functional Rot of $17 \mathrm{kDa}$ in the various isogenic $S$. aureus strains. The published $S$. aureus $\mathrm{N} 315$ genome reveals at least ten proteins with significant homology to SarA (Kuroda et al., 2001), of which nine have been characterized. Recently, we determined the length of SarZ (SA2174 of S. aureus N315) by primer extension and found it to be 148 residues (unpublished data) as predicted in the published S. aureus genomes. Therefore, the lengths of all ten SarA paralogues, including Rot as determined in this report, are known. An alignment of all ten SarA paralogues, including the 133residue Rot, with other SarA paralogues is much more ordered and of higher degree than the alignment of the 166or 153-residue counterparts.

\section{Analysis of rot transcription in wild-type RN6390 and in an assortment of isogenic mutants}

Northern blot assays were performed with a rot probe (425 bp within the coding region) with the various regulatory mutants in our collection to determine if the transcription of the rot gene is affected by inactivation of other regulatory systems, including those of the sarA family of genes. Results demonstrated that the level of rot transcript was increased only in the sarA and sarS mutants, whereas no significant changes were observed in other mutants at the post-exponential phase of growth (Fig. 3a). A diffused pattern of transcripts appeared particularly in the lateexponential and post-exponential phases, probably due to the presence of the multiple transcripts as evidenced in the primer extension experiment. Alternatively, it may have been due to cleavage of the rot transcripts by the base pairing of the agr RNAIII molecule as suggested by Geisinger et al. (2006) or the degradation of the rot transcripts at the postexponential phase of growth. In addition, it is consistent with the primer extension results that the sarA gene product acts as a repressor for rot transcription.

Similarly, we performed Northern blot analysis to determine the variation of rot transcription within the various growth phases by isolating total RNA from different time points in various isogenic strains, as shown in Fig. 3(b) and (c). Maximum expression of rot transcription was observed during the exponential phase of growth (between 3 and $4 \mathrm{~h}$ ) in the wild-type strain, whereas the transcription decreased during the early and post-exponential phase of growth. The inactivation of sarA yielded a significantly higher level of rot transcription in all phases of growth, whereas the introduction of a single copy of the sarA gene into the lipase locus ( $g e h$ ) of the respective sarA mutant yielded expression of rot transcripts to near-parental levels, suggesting that the expression of rot is truly repressed by the sarA gene product. Nevertheless, the cause of or reason for the apparent discrepancy in the expression of rot transcripts (less for sarA mutant at $3 \mathrm{~h}$, higher for sarA-complemented sarA strain at $4 \mathrm{~h}$, or less for agr mutant at $5 \mathrm{~h}$ ) is unknown. This observation is consistent with the repeated experiments.

These results were also confirmed by promoter fusion studies using a $317 \mathrm{bp}$ DNA fragment (positioned from 1 to 317; Fig. 2) containing all four promoters of the rot gene with a promoterless $g f p_{u v r}$ reporter gene in the various isogenic strains of RN6390. A substantial increase in mean GFP fluorescence for the sarA mutant as compared to that for the wild-type RN6390 and the single-copy complemented sarA mutant was found as shown in Fig. 4. However, the twofold increment of mean GFP activity in the sarS mutant is probably due to the indirect effect, which is yet to be verified by performing more confirmative experiments. We consistently observed a partial decrease or increase in rot promoter fusion activity in some of the mutants, which we speculate might be due to a direct or an indirect effect, which is yet to be verified.

\section{Binding of SarA protein to the rot promoter region}

The data presented in earlier sections clearly suggest that expression of rot transcription was affected only by sarA mutation among the mutants tested; we therefore speculated that SarA might bind to the rot promoter region to repress rot transcription. To test this, we employed the 317 bp rot promoter fragment used in transcriptional fusion 
(a)

\begin{tabular}{|c|c|c|c|c|c|c|c|c|c|c|c|}
\hline$\xi$ & ర్లా & $\begin{array}{l}\mathbb{్} \\
\mathbb{్}\end{array}$ & $\underset{\mathbb{W}}{\mathbb{్}}$ & $\underset{\mathscr{\varpi}}{\mathscr{W}}$ & $\underset{\mathscr{W}}{\leftarrow}$ & ج్ల్ల & 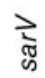 & 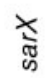 & స్ & $\underset{\mathscr{W}}{\mathbb{E}}$ & $\frac{n}{5}$ \\
\hline 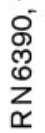 & 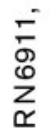 & 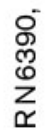 & 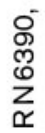 & 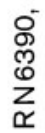 & 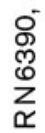 & 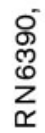 & 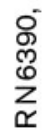 & 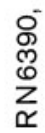 & 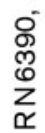 & $\begin{array}{l}\mathcal{D}^{-} \\
\text {ల్ } \\
\underset{\sim}{Z}\end{array}$ & 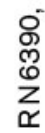 \\
\hline
\end{tabular}

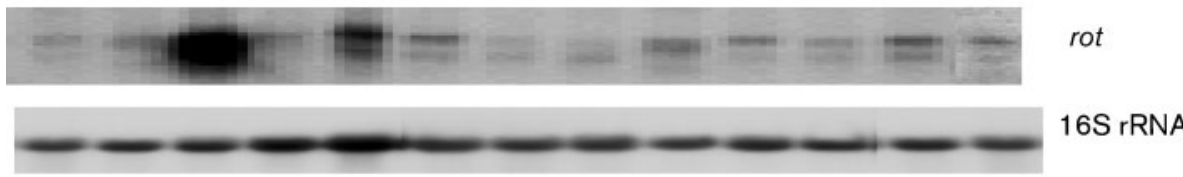

(b)

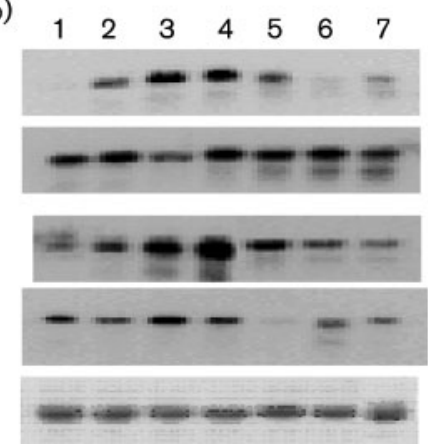

h

RN6390, wt

A L C488, sarA

ALC812, sar $A$

/cpsarA

RN6911, agr

$16 \mathrm{~S}$ rRNA (c)

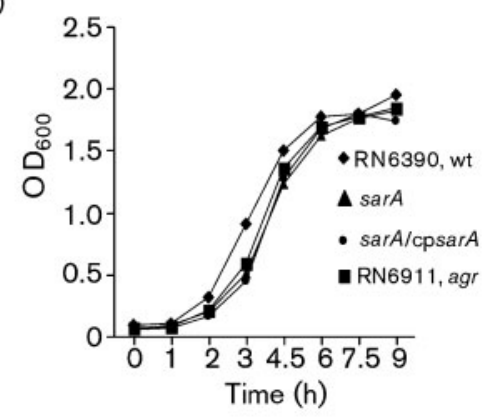

Fig. 3. Northern analysis of the rot transcript in the wild-type, various isogenic mutants, and single-copy-complemented strain (cp) at post-exponential phase $\left(\mathrm{OD}_{600} \sim 1.7\right)$ of growth in (a), various time points of growth in (b) and growth curves of the wild-type RN6390, RN6390 sarA mutant, complemented sarA mutant and RN6911 agr mutant strains in (c). A 425 bp DNA fragment containing the rot gene was used for hybridization. The $723 \mathrm{bp}$ internal fragment of $16 \mathrm{~S}$ rDNA was used for hybridization as a loading control in (a) and (b). The $\mathrm{OD}_{600}$ of various cultures was measured in a Spectronic 20 spectrophotometer. Note that the time scale in (c) is non-linear.

studies for DNA-binding assay. The $\gamma_{-}{ }^{32} \mathrm{P}$-radiolabelled DNA fragment was used in gel shift assays with varying amounts of purified SarA protein (Fig. 5). A retarded

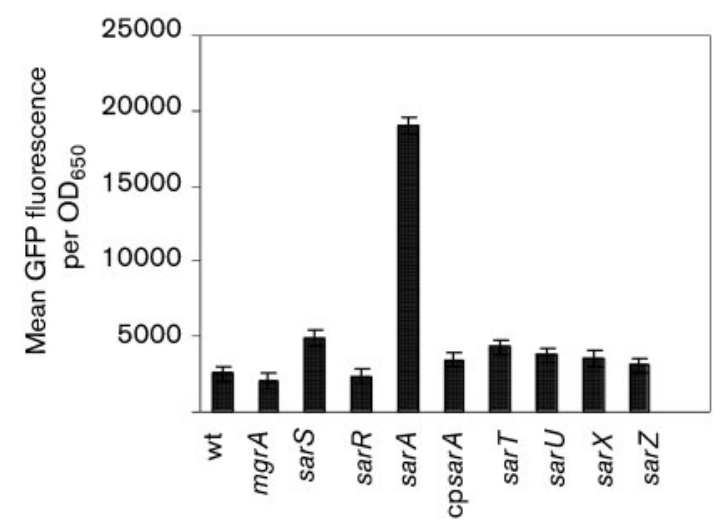

Fig. 4. Promoter expression of the combined rot promoter region fused to a $g f p_{u v r}$ reporter gene in the wild-type and various isogenic mutants at the post-exponential $(7 \mathrm{~h})$ phase of growth. To minimize variations in fluorescence attributable to cell density, the data are presented as the mean of triplicate samples in fluorescence units per $\mathrm{OD}_{650}$.
DNA-protein complex was detected with as little as $0.2 \mu \mathrm{g}$ SarA. The retarded protein-DNA complex band became predominant with an increasing concentration of SarA protein, with complete conversion occurring at $\sim 0.5 \mu \mathrm{g}$. The unlabelled promoter fragment competed effectively with labelled fragment, whereas an unlabelled non-specific DNA fragment (185 bp PsarX fragment; Manna \& Cheung, 2006b) did not compete significantly with the radiolabelled fragment, suggesting that the DNA-protein interaction was specific.

Previously, we mapped the consensus DNA-binding sequences of the SarA protein to upstream of various target promoter regions based on DNase I footprinting, and we found SarA consensus binding sequences to be $5^{\prime}$ ATTTgTATtTAATATTTataTAATT-3' (Chien \& Cheung, 1998; Chien et al., 1999; Manna et al., 2004; Manna \& Cheung, 2006a). In aligning the 26 bp SarA consensus binding sequences with the upstream rot promoter region, we found four matching regions. Of these four matching regions, three were in top-strand positions 60-84 (B1), 86-110 (B2) and 174-198 (B3), and one was in the bottomstrand position 113-88 (B4) (Fig. 2). Of the 26 nucleotides, $18,17,19$ and 16 matched, respectively. The location of SarA-binding sites within the P2, P3 and P4 promoter regions is consistent with the results of the primer extension 


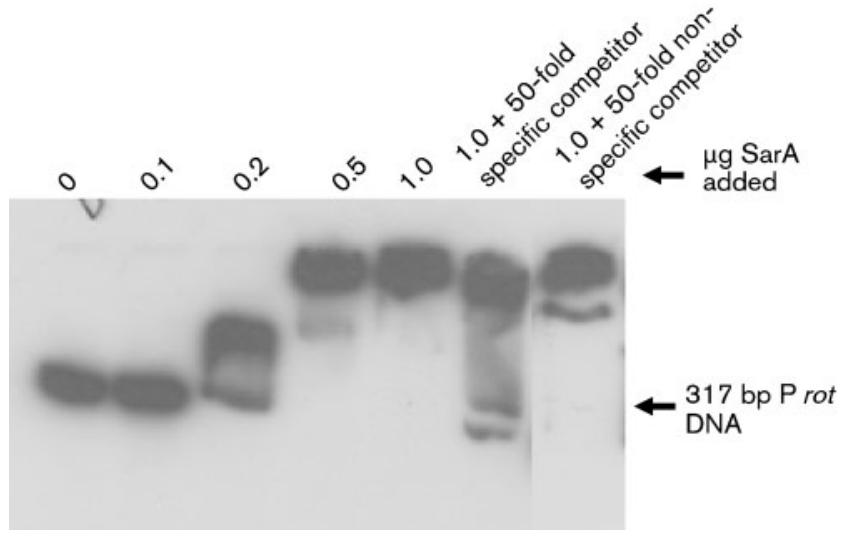

Fig. 5. Autoradiogram of an $8.0 \%$ polyacrylamide gel showing gel shifts for purified SarA protein with a $\gamma^{32} \mathrm{P}$-labelled, $317 \mathrm{bp}$ rot promoter fragment. Mobility of the DNA band in the presence of increasing amounts of SarA protein, as indicated on the top. Cold competition lanes with 50-fold excess (molar ratio) of unlabelled $317 \mathrm{bp}$ rot fragment (specific) and $185 \mathrm{bp}$ sarX promoter fragment (non-specific; Manna \& Cheung, $2006 \mathrm{~b})$ are indicated and contained $1.0 \mu \mathrm{g}$ of the purified SarA protein.

and Northern blot analyses of the sarA mutant as compared to the wild-type strain.

\section{DISCUSSION}

The fact that $S$. aureus exhibits complex patterns of protein expression in response to various environmental conditions (Arvidson \& Tegmark, 2001; Cheung \& Zhang, 2002; Lowy, 1998; Novick, 2003; Projan \& Novick, 1997) is consistent with the notion that large numbers of complex regulatory systems coordinate expression of most virulence factors that are involved in the infectious processes. The transcriptional regulators, particularly the SarA protein family, are emerging as an important regulatory system for potential interference and therefore control of S. aureus infections. In prior studies, we and others have characterized nine SarA paralogues, including SarA, SarR, SarS, Rot, SarT, SarU, MgrA, SarV and SarX, all of which are involved in regulation of both regulatory genes including the sarA family of genes, and virulence genes in S. aureus.

The predicted lengths of the 10 major SarA paralogues in $S$. aureus genomes are consistent with the lengths determined by primer extension, with the exception of the SarX and Rot proteins. The predicted length of Rot was reported in 11 published $S$. aureus genomes to be 166 residues (S. aureus strains RF122, COL, 252, 476, USA300), 153 residues ( $S$. aureus strains JH1, JH9, MW2, Mu50, N315), or 133 residues ( $S$. aureus strain NCTC 8325), but our various experimental analyses, along with data of Oscarsson et al. (2005) and Geisinger et al. (2006), indicate that the length of Rot is likely to be 133 residues. The Rot protein is the tenth member of the SarA protein family found to have low sequence homology with SarA protein ( $e$-value of 0.003 with $25 \%$ identity and $50 \%$ similarity), but the degree of conserved residues throughout the entire length of all paralogues is significant. Interestingly, many basic residues are conserved within members of the SarA protein family, consistent with the notion that the DNA-binding domains are highly conserved, and detailed mutational analysis of SarR and SarA indicated that many of the conserved basic residues (e.g. K52, K80, R82, R88, etc., of SarR) are indeed involved in DNA binding (Liu et al., 2006; Manna \& Cheung, 2006a).

The transcription of sarA-like genes is highly variable in various S. aureus strains including strain RN6390. For instance, sarA, sarR, $m g r A$ and sarX transcripts are highly expressed, whereas the transcripts of $\operatorname{sar} U$, sarT, sarV and sarS are not readily detectable under normal laboratory growth conditions, probably due to the repression of other regulatory gene products. We found the rot gene to be poorly transcribed in the early (lag) and post-exponential phases of growth, whereas transcription was significantly higher in the exponential phase in the wild-type strain. This finding is consistent with the earlier observation that the rot gene is transcribed maximally in the exponential phase of growth (McNamara et al., 2000). Northern analysis of rot transcription in mutants of agr, sae, sigB and nine sarA family genes revealed that transcription of rot remains unaltered in these mutants at the post-exponential phase of growth, with the exception of sarA and sarS. Rot transcription is increased in the sarA mutant and is nearly restored upon single-copy complementation of the sarA gene. Similar results were observed with a combined rot promoter $g f p_{u v r}$ fusion. However, rot transcription was increased nearly twofold in the sarS mutant as determined from Northern and transcriptional fusion assays, probably due to an indirect effect of sarS. Alternatively, we are not ruling out the direct involvement of sarS, which has yet to be established. Regulation of the sarA family of genes is intertwined in nature (Manna \& Cheung, 2006a, b). It should be stressed that sarS is transcribed in the early growth phase and tapers off in transcription at the postexponential and stationary phases of growth in RN6390 strain (Cheung et al., 2001; Tegmark et al., 2000). In the future, we will be analysing various sarA family mutant strains of $S$. aureus from different genetic backgrounds to determine if there is any variability of the data presented in this report.

In the primer extension experiment, we found that four transcripts encoding the major Rot polypeptide originated from different locations. The abolished transcription of the P2 and P3 transcripts and reduced levels of the P1 and $\mathrm{P} 4$ transcripts in the wild-type strain, as compared to the sarA mutant, clearly indicated that SarA is involved in negative regulation of rot transcription. The pattern of rot transcription in various growth phases does not correlate with the expression of the SarA protein in S. aureus as 
SarA is expressed more in the exponential than in the post-exponential phase of growth (Manna \& Cheung, 2001). This means that SarA may not be solely involved in repression of rot transcription, which may interact with one or more other intermediary transcription factors, probably with Rot, as it has been suggested that Rot may act as an autorepressor (Geisinger et al., 2006). We believe the regulation of the target genes by the SarA paralogues is complex, and may involve more than one protein. The details of the SarA-mediated rot transcription remain to be determined. It should be noted that the involvement of the agr RNAIII molecule in the regulation of rot expression at the translational level (Geisinger et al., 2006) has been reported.

Utilizing gel shift studies, we found that purified SarA binds to the rot promoter region with high affinity. Four SarA consensus binding sequences were found within the $350 \mathrm{bp}$ rot promoter region, three (B1-3) on the top strand and one (B4) on the complementary strand of the B2 region. B1, B2 and $\mathrm{B} 4$ overlapped within the $\mathrm{P} 4$ and $\mathrm{P} 3$ promoter regions, whereas the $\mathrm{B} 3$ site was located near the -10 region of the $\mathrm{P} 2$ promoter. We speculate that on the B3 site SarA probably interacts with the other SarA dimeric molecules at the B1, $\mathrm{B} 2$, or B4 locations to loop out the DNA, thus interfering with binding of RNA polymerase to the $\mathrm{P} 2$ and partially the P1 promoters. Although the mechanisms by which SarA regulates its target genes are not well understood, we now know that more than two consensus-binding sites are present in the promoter regions of most of the SarAregulated target genes analysed, including the agr locus (Manna \& Cheung, 2006a). These results are consistent with our earlier finding that most of the SarA paralogues regulate each other. Furthermore, the SarA paralogues involved in virulence gene regulation are intertwined: for example, sarR represses SarA expression (Manna \& Cheung, 2001), sarA represses and rot activates sarS expression (Cheung et al., 2001; Said-Salim et al., 2003; Tegmark et al., 2000), sarA negatively regulates sarT and sarT activates sarS (Schmidt et al., 2001), both SarA and MgrA repress sarV (Manna et al., 2004), while MgrA activates sarX transcription (Manna \& Cheung, 2006b).

To further define the precise function of each of the SarA paralogues, we are in the process of analysing the effects of various combined knockouts of different sarA family genes on other regulatory genes, and investigating the molecular interaction of SarA paralogues with the upstream region of the target genes.

\section{ACKNOWLEDGEMENTS}

We thank Ambrose Cheung for the use of his laboratory to conduct some parts of experiments described in this manuscript. We would also like to thank Leigh Washburn for critical reading and helpful comments on the manuscript. This work was supported by 2010initiative start-up and SD BRIN funds awarded to A. C. M.

\section{REFERENCES}

Arvidson, S. \& Tegmark, K. (2001). Regulation of virulence determinants in Staphylococcus aureus. Int J Med Microbiol 291, 159-170.

Ausubel, F. M., Brent, R., Kingston, R. E., Moore, D. D., Seidman, J. G., Smith, J. A. \& Struhl, K. (2000). Current Protocols in Molecular Biology. New York: Wiley.

Bayer, M. G., Heinrichs, J. H. \& Cheung, A. L. (1996). The molecular architecture of the sar locus in Staphylococcus aureus. J Bacteriol 178, 4563-4570.

Blevins, J. S., Gillaspy, A. F., Rechtin, T. M., Hurburt, B. K. \& Smeltzer, M. S. (1999). The staphylococcal accessory regulator (sar) represses transcription of the $S$. aureus collagen adhesin gene (cna) in an $a g r$-independent manner. Mol Microbiol 33, 317-326.

Boyce, J. M. (1997). Epidemiology and prevention of nosocomial infections. In The Staphylococci in Human Diseases, vol. 12, pp. 309-329. Edited by K. B. Crossley \& G. L. Archer. New York: Churchill Livingstone.

Cheung, A. L. \& Zhang, G. (2002). Regulation of virulence determinants in Staphylococcus aureus by the SarA protein family. Front Biosci 7, D1825-D1842.

Cheung, A. L., Koomey, J. M., Butler, C. A., Projan, S. J. \& Fischetti, V. A. (1992). Regulation of exoptotein expression in Staphylococcus aureus by a locus (sar) distinct from agr. Proc Natl Acad Sci U S A 89, 6462-6466.

Cheung, A. L., Schmidt, K. A., Bateman, B. \& Manna, A. C. (2001). SarS, a SarA homolog repressible by $a g r$, is an activator of protein A synthesis in Staphylococcus aureus. Infect Immun 69, 2448-2455.

Chien, Y.-t. \& Cheung, A. L. (1998). Molecular interactions between two global regulators, sar and agr, in Staphylococcus aureus. J Biol Chem 273, 2645-2652.

Chien, Y.-t., Manna, A. C., Projan, S. J. \& Cheung, A. L. (1999). SarA, a global regulator of virulence determinants in Staphylococcus aureus, binds to a conserved motif essential for sar-dependent gene regulation. J Biol Chem 274, 37169-37176.

Dunman, P. M., Murphy, E., Haney, S., Palacios, D., Tucker-Kellogg, G., Wu, S., Brown, E. L., Zagursky, R. J., Shlaes, D. \& Projan, S. J. (2001). Transcriptional profiling-based identification of Staphylococcus aureus genes regulated by agr and/or sarA loci. J Bacteriol 183, 7341-7353.

Geisinger, E., Adhikari, R. P., Jin, R., Ross, H. F. \& Novick, R. P. (2006). Inhibition of rot translation by RNAIII, a key feature of $a g r$ function. Mol Microbiol 61, 1038-1048.

Kahl, B., Goulian, M., Van Wamel, W., Herrmann, M., Simon, S., Kaplan, G., Peters, G. \& Cheung, A. L. (2000). Staphylococcus aureus RN6390 replicates and induces apoptosis in a pulmonary epithelial cell line. Infect Immun 68, 5385-5392.

Kuroda, M., Ohta, T., Uchiyama, I., Baba, T., Yuzawa, H., Kobayashi, I., Cui, L., Oguchi, A., Aoki, K. I. \& other authors (2001). Whole genome sequencing of methicillin-resistant Staphylococcus aureus. Lancet 357, 1225-1240.

Liu, Y., Manna, A., Li, R., Martin, W. E., Murphy, R. C., Cheung, A. L. \& Zhang, G. (2001). Crystal structure of the SarR protein from Staphylococcus aureus. Proc Natl Acad Sci U S A 98, 6877-6882.

Liu, Y., Manna, A. C., Pan, C.-H., Kriksunov, I. A., Thiel, D. J., Cheung, A. L. \& Zhang, G. (2006). Structural and functional analyses of the global regulatory protein SarA from Staphylococcus aureus. Proc Natl Acad Sci U S A 103, 2392-2397.

Lowy, F. (1998). Staphylococcus aureus infections. N Engl J Med 339, $520-532$.

Luong, T. T., Newell, S. W. \& Lee, C. Y. (2003). $m g r$, a novel global regulator in Staphylococcus aureus. J Bacteriol 185, 3703-3710. 
Manna, A. \& Cheung, A. L. (2001). Characterization of sarR, a modulator of sar expression in Staphylococcus aureus. Infect Immun 69, 885-896.

Manna, A. C. \& Cheung, A. L. (2003). sarU, a sarA homolog, is repressed by SarT and regulates virulence genes in Staphylococcus aureus. Infect Immun 71, 343-353.

Manna, A. C. \& Cheung, A. L. (2006a). Transcriptional regulation of the agr locus and the identification of DNA-binding residues of the global regulatory protein SarR in Staphylococcus aureus. Mol Microbiol 60, 1289-1301.

Manna, A. C. \& Cheung, A. L. (2006b). Expression of SarX, a negative regulator of agr and exoprotein synthesis, is activated by MgrA in Staphylococcus aureus. J Bacteriol 188, 4288-4299.

Manna, A. C., Ingavale, S. S., Maloney, M., van Wamel, W. \& Cheung, A. L. (2004). Identification of $\operatorname{sarV}$ (SA2062), a new transcriptional regulator, is repressed by SarA and MgrA (SA0641) and involved in the regulation of autolysis in Staphylococcus aureus. J Bacteriol 186, 5267-5280.

McNamara, P. J. \& Bayer, A. S. (2005). A rot mutation restores parental virulence to an agr-null Staphylococcus aureus strain in a rabbit model of endocarditis. Infect Immun 73, 3806-3809.

McNamara, P. J., Milligan-Monroe, K. C., Khalili, S. \& Proctor, R. A. (2000). Identification, cloning, and initial characterization of rot, a locus encoding a regulator of virulence factor expression in Staphylococcus aureus. J Bacteriol 182, 3197-3203.

Novick, R. P. (2003). Autoinduction and signal transduction in the regulation of staphylococcal virulence. Mol Microbiol 48, 1429-1449.

Oscarsson, J., Harlos, C. \& Arvidson, S. (2005). Regulatory role of proteins binding to the spa (protein A) and sarS (staphylococcal accessory regulator) promoter regions in Staphylococcus aureus NTCC 8325-4. Int J Med Microbiol 295, 253-266.
Projan, S. J. \& Novick, R. P. (1997). The molecular basis of pathogenicity. In The Staphylococci in Human Diseases, pp. 55-81. Edited by K. B. Crossley \& G. L. Archer. New York: Churchill Livingstone.

Rechtin, T. M., Gillaspy, A. F., Schumacher, M. A., Brennan, R. G., Smeltzer, M. S. \& Hurlburt, B. K. (1999). Characterization of the SarA virulence gene regulator of Staphylococcus aureus. Mol Microbiol 33, 307-316.

Said-Salim, B., Dunman, P. M., McAleese, F. M., Macapagal, D., Murphy, E., McNamara, P. J., Arvidson, S., Foster, T. J., Projan, S. J. \& Kreiswirth, B. N. (2003). Global regulation of Staphylococcus aureus genes by Rot. J Bacteriol 185, 610-619.

Schenk, S. \& Laddaga, R. A. (1992). Improved methods for electroporation of Staphylococcus aureus. FEMS Microbiol Lett 94, 133-138.

Schmidt, K. A., Manna, A. C., Gill, S. \& Cheung, A. L. (2001). SarT, a repressor of alpha-hemolysin in Staphylococcus aureus. Infect Immun 69, 4749-4758.

Tegmark, K., Karlsson, A. \& Arvidson, S. (2000). Identification and characterization of SarH1, a new global regulator of virulence gene expression in Staphylococcus aureus. Mol Microbiol 37, 398-409.

Tormo, M. A., Marti, M., Valle, J., Manna, A. C., Cheung, A. L., Lasa, I. \& Penades, J. R. (2005). SarA is an essential positive regulator of Staphylococcus epidermidis biofilm development. J Bacteriol 187, 2348-2356.

Truong-Bolduc, Q. C., Zhang, X. \& Hooper, D. C. (2003). Characterization of NorR protein, a multifunctional regulator of norA expression in Staphylococcus aureus. J Bacteriol 185, 3127-3138.

Tseng, C. W. \& Stewart, G. C. (2005). Rot repression of enterotoxins B expression in Staphylococcus aureus. J Bacteriol 187, 5301-5309.

Edited by: W. B. van Leeuwen 